\title{
Demonstration of the combination of slice imaging and Rydberg tagging for studies of photodissociation dynamics
}

\author{
H. A. Cruse and T. P. Softley \\ Department of Chemistry, Chemistry Research Laboratory, University of Oxford, Mansfield Road, \\ Oxford OX1 3TA, United Kingdom
}

(Received 8 April 2004; accepted 15 June 2004)

\begin{abstract}
The slice-imaging variant of photofragment ion imaging is combined with Rydberg tagging. The photodissociation of $\mathrm{NO}_{2}$ at $355 \mathrm{~nm}$ is used as the test system and the NO fragments are Rydberg tagged by two-photon two-color excitation via the intermediate $A^{2} \Sigma^{+}$state. Images obtained by this method are compared with ion images obtained in the same apparatus using the approach of Kitsopoulos and co-workers [Rev. Sci. Instrum. 72, 3848 (2001)]. Comparable resolution and angular distributions are obtained in the two cases. It is proposed that the method demonstrated here could provide a complementary approach to existing ion-imaging methods, especially where resonantly enhanced multiphoton ionization detection of fragments is problematic.

(c) 2004 American Institute of Physics. [DOI: 10.1063/1.1779621]
\end{abstract}

\section{INTRODUCTION}

In this paper we report the experimental demonstration of the combination of two techniques recently developed for the study of photodissociation dynamics, namely, (i) molecular Rydberg tagging and (ii) the slice-imaging variant of photofragment ion imaging. Ion-imaging techniques have been used to great advantage in the measurement of photofragment kinetic energies and angular distributions for a range of photodissociation processes, ${ }^{1}$ and have also been applied to bimolecular collisional processes. ${ }^{2}$ The introduction of velocity mapping has greatly enhanced the energy resolution available. ${ }^{3}$ In a typical ion-imaging experiment the quantity measured is the two-dimensional projection of the photofragment velocity distribution on to the detector; in the majority of cases the inverse Abel transformation is used to reconstruct an infinitesimally thin slice through the center of the three-dimensional distribution from this information. However, the Abel transform process introduces artificial noise into the data, particularly along the centerline, and it can only be used for an experimental geometry with an axis of cylindrical symmetry parallel to the plane of the imaging detector. Furthermore, the Abel transform is only a valid reconstruction method if the entire photofragment distribution is captured on the detector. Other reconstruction techniques have been devised to overcome these limitations, ${ }^{4-6}$ but each has its own drawbacks. As a result several experimental schemes have been developed which directly measure the center slice of the photofragment distribution. ${ }^{7-10}$ One of the most successful has been "slice imaging," which was introduced by Kitsopoulos and co-workers in 2001, ${ }^{7}$ and incorporates some elements of the velocity-mapping technique.

The fundamental experimental distinction between slice imaging and conventional velocity mapping is that a delay is introduced between the ionization of photofragments and their extraction. In this time the ion cloud is allowed to expand in a field-free region and the resultant spatial extent of the fragment sphere at the time of extraction is converted into a spread of arrival times at the detector of the order of 500 ns. Fast gating of the detector at the required time of flight yields a slice of the elongated fragment distribution, and the central slice is equivalent to the Abel inversion of an image obtained using the velocity-mapping technique.

The main advantage of the slice-imaging technique is that only the center slice of the three-dimensional photofragment distribution is measured and analyzed. Thus, velocity distributions may be obtained directly from the raw image without the use of a reconstruction method. Furthermore, it is not essential to record the entire image, with the result that extraction and focusing voltages may be chosen to zoom in on the slowest fragments and detect them with the highest possible resolution. Therefore, slice imaging is ideally suited to the study of fragments with low recoil velocities, such as those formed in near-threshold photodissociation or those with a cofragment of light mass, e.g., as demonstrated in studies of slow $\mathrm{Br}$ fragments from the photodissociation of $\mathrm{HBr}^{11}$

The exploitation of Rydberg-tagging methods in imaging studies of photodissociation dynamics is a relatively new area of research, ${ }^{12-14}$ and can be considered as a development of the H-atom Rydberg tagging employed in translational energy spectroscopy. ${ }^{15}$ In the Rydberg-tagging approach, the nascent photofragments are not immediately ionized but are excited to high- $n$ Rydberg states, and the fragment cloud continues to expand as a bunch of neutral molecules. After a time delay, a delayed extraction pulse is applied to field ionize the Rydberg states and accelerate the resultant ions on to an imaging detector. In principle this method offers several advantages over conventional ion imaging based on resonantly enhanced multiphoton ionization (REMPI) excitation of the nascent fragments: First, in situations where there is a high density of ions in the excitation region produced by REMPI, Coulomb explosion may distort ion images. This effect is particularly pronounced when probing fragments with low recoil velocities, such as those 
formed in near-threshold photolysis. Coulomb explosion can be eliminated by tagging the molecules as neutral Rydberg states. Second, it is anticipated that the resolution of ion images will eventually be limited by the recoil velocity of the electron. ${ }^{16,17}$ This restriction is removed by using field ionization and extraction of Rydberg-tagged fragments, for which the electron recoil is effectively zero. Third, many molecules of interest, such as $\mathrm{CH}_{2}, \mathrm{C}_{2} \mathrm{H}$, and $\mathrm{CO}_{2}$, have no suitable intermediate for resonant multiphoton ionization. However, state-selective detection can be achieved in these cases by using vacuum ultraviolet sources to excite directly to high- $n$ Rydberg states, as demonstrated by the one-photon zero-kinetic-energy (ZEKE) spectrum of $\mathrm{CH}_{2}$ recorded by Merkt and co-workers. ${ }^{18}$ Consequently, imaging of Rydbergtagged fragments can provide a solution in cases where ion imaging is impracticable, thus expanding the range of chemical systems that can be studied. Finally, we recently introduced the concept of utilizing the properties of high- $n$ Rydberg states in inhomogeneous electric fields to probe selected regions of the photofragment distribution. ${ }^{13}$ By discriminating against energetic fragments this technique can be used to focus on the threshold region, and so enhance understanding of near-threshold dynamics. In principle, the implementation of carefully designed field configurations will enable in situ probing of any position within the photofragment distribution.

However, pulsed-field-ionization (PFI) methods appear to be incompatible with the velocity-mapping technique as it was originally conceived, ${ }^{3}$ and this has limited the resolution obtainable in Rydberg-tagging/ion-imaging experiments. In a standard pulsed-field-ionization experimental setup, ${ }^{12}$ excitation to high- $n$ Rydberg states occurs between two fine-mesh grids and at the time of excitation a small, uniform electric field is applied in this region to repel ions formed by direct ionization or autoionization. Once the spatial separation of ions and Rydberg states has been achieved, an extraction field is applied which field ionizes the Rydberg states and accelerates all the ionized fragments towards the detector. In this scheme, spatial separation before extraction is converted to separation in time of flight at the detector, which is gated to detect only Rydberg states.

It can be seen that two essential components of a successful PFI experiment are the capacity to apply uniform discrimination fields at the point of excitation and the facility to introduce a low-field delay between excitation and extraction. Velocity-mapping ion optics have open apertures, and so they do not provide a well-defined uniform field region, and a delay before extraction would merely serve to reduce the effectiveness of the mapping, since the effective photofragment source size is increased. However, the sliceimaging technique naturally includes both the features required for pulsed-field ionization and therefore provides a route to obtain high-resolution images of Rydberg-tagged photofragments.

The aim of the work described in this paper is to demonstrate that slice imaging can be used to record angular and energy distributions of Rydberg-tagged photodissociation products, and thus to combine the advantages of this highresolution imaging technique with the versatility of Rydberg-

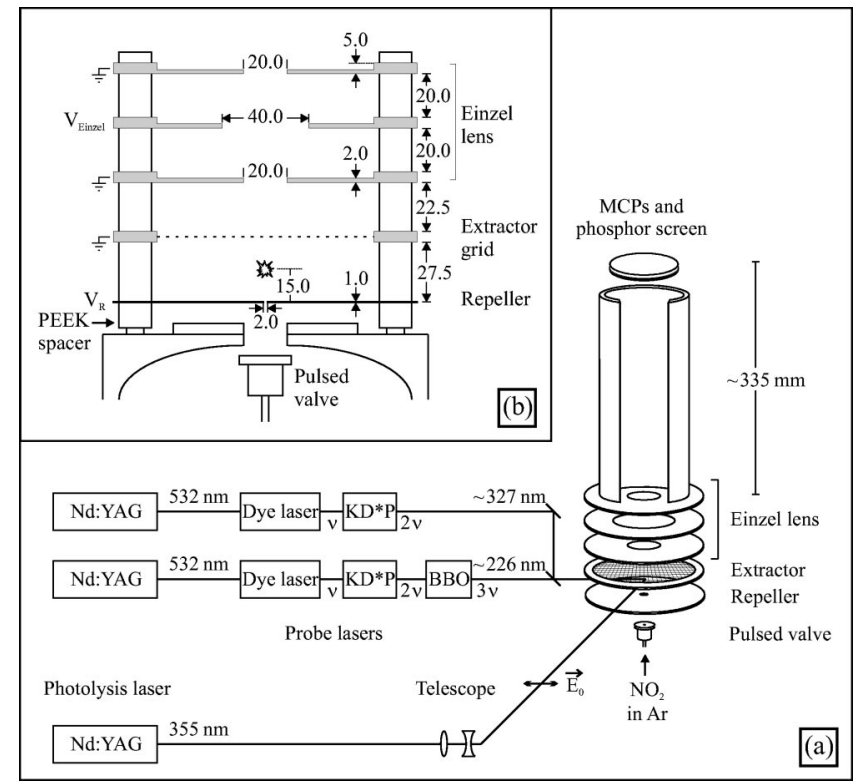

FIG. 1. (a) Schematic diagram of the experimental arrangement for the slice-imaging of Rydberg-tagged $\mathrm{NO}$ fragments from $\mathrm{NO}_{2}$ photodissociation. (b) Schematic diagram of the electrode arrangement used for the sliceimaging studies in this work (all dimensions in millimeter).

state experiments. The photodissociation of $\mathrm{NO}_{2}$ is chosen as a test system with which to characterize this technique. This efficient, extensively studied dissociation process yields NO fragments which can be excited to stable high- $n$ Rydberg states with the long lifetimes necessary to survive until detection. Conventional slice images of NO ions, produced by REMPI excitation of the NO fragments, are recorded using the same experimental apparatus to provide a direct comparison with the performance of the Rydberg-tagging/sliceimaging technique.

\section{EXPERIMENT}

The spectroscopic schemes used in this work for photodissociation and Rydberg tagging are broadly similar to those utilized in Ref. 12. In brief, a pulsed supersonic free-jet expansion of $\mathrm{NO}_{2}$ is intersected by a photodissociation laser of wavelength $355 \mathrm{~nm}$. Excitation to the $\widetilde{A}^{2} B_{2}$ state is followed by fast internal conversion and dissociation to $\mathrm{O}\left({ }^{3} P\right)+\mathrm{NO}$ on the ground-state surface. For the Rydberg-tagging experiments (Sec. III B) high- $n$ Rydberg states of the ensuing NO photofragments are populated using a $\left(1+1^{\prime}\right)$ probing scheme via the $A^{2} \Sigma^{+}$intermediate state. Prompt ions are repelled by a small uniform field applied in the excitation region whereas Rydberg states are field ionized by a delayed extraction pulse and accelerated towards the detector. For the REMPI-detection experiments (Sec. III A) the second probe laser is tuned above the ionization threshold to produce $\mathrm{NO}^{+}$ ions directly.

The experimental apparatus is represented schematically in Fig. 1. Prior to use, $\mathrm{NO}_{2}$ (BOC gases, $\mathrm{CP}$ grade) is stored with oxygen $\left[p\left(\mathrm{NO}_{2}\right): p\left(\mathrm{O}_{2}\right)=1: 5\right]$ for $12 \mathrm{~h}$ to reduce the concentration of residual $\mathrm{NO}$ in the sample. Purified $\mathrm{NO}_{2}$, seeded in Ar at 5\% molar fraction, is expanded into the vacuum chamber through a pulsed nozzle (General Valve, 1 
$\mathrm{mm}$ orifice, $400 \mu$ s pulse duration at $10 \mathrm{~Hz}$ ), at a backing pressure of 3 bars. The pressure of the experimental chamber is maintained at $2 \times 10^{-6}$ mbar during data acquisition.

The photodissociation source is provided by the $355 \mathrm{~nm}$ output of a Nd:YAG laser (Spectra Physics GCR-190), attenuated to $\sim 10 \mathrm{~mJ}$ per pulse $(10 \mathrm{~Hz})$. The beam diameter is reduced to $\sim 2 \mathrm{~mm}$ using a telescope and the polarization direction of this laser is aligned parallel to the detector face. For both the $\left(1+1^{\prime}\right)$ Rydberg tagging and REMPI schemes, the first wavelength ( $\lambda_{1} \sim 226 \mathrm{~nm}, 100 \mu \mathrm{J}$ per pulse) is provided by the frequency-tripled output of a Nd:YAG-pumped dye laser (Spectron Laser Systems, SL803+SL4000G) operating at $\sim 678 \mathrm{~nm}$ in a 2:1 LDS-698/DCM dye mixture in methanol. Excitation to either the continuum (REMPI detection) or a high- $n$ Rydberg state (PFI detection) is achieved using the frequency-doubled output $\left(\lambda_{2} \sim 327 \mathrm{~nm}, 2 \mathrm{~mJ}\right.$ per pulse) of a second Nd:YAG-pumped dye laser operating between 653 and $655 \mathrm{~nm}$ in DCM dye in methanol. The fundamental output of each dye laser is calibrated using a wave meter (Burleigh WA5500). The probe lasers are combined on a dichroic mirror and are introduced coaxially into the chamber, perpendicular to both the photolysis laser and the detection axis, and are delayed by $\approx 15 \mathrm{~ns}$ and $30 \mathrm{~ns}$ with respect to the photolysis laser. The polarizations of both lasers are vertical and thus perpendicular to the plane of the detector, and the probe beam diameters at the point of intersection of the free-jet expansion are $2 \mathrm{~mm}$ and $4 \mathrm{~mm}$ for lasers 1 and 2, respectively.

Figure 1(b) shows the configuration of the slice-imaging ion optics. The free-jet expansion enters the interaction region through a $2 \mathrm{~mm}$ aperture in the repeller plate and is intersected by the laser beams at a point midway between the repeller plate and the extractor grid (Buckbee Mears mesh, 90 lines per in.). After excitation the fragment cloud is allowed to expand in either a field-free region (ion detection) or a small uniform field (Rydberg-state detection) for 4-5 $\mu \mathrm{s}$, after which time a high-voltage switch (Behlke, $10 \mathrm{~ns}$ rise time) is used to apply an extraction voltage of order $1000 \mathrm{~V}$ to the repeller electrode. The resulting field serves to field ionize the Rydberg states and accelerates the ion cloud towards the detector. As a result of the spatial distribution of the cloud at the time of extraction, the ions that are back scattered with respect to the molecular beam direction remain in the high-field region for longer than the corresponding forward-scattered ions, and experience a greater acceleration. Therefore, as the ion cloud moves towards the detector its spatial extent along the time-of-flight axis, $\Delta x$, decreases to zero and then becomes greater again, as the backscattered ions overtake the portion of the ion cloud which was originally forward scattered. $V_{\mathrm{R}}$ is chosen such that the spread in arrival times at the detector is of the order of several microseconds. A dual microchannel plate (MCP) and phosphorscreen detection system is used. Narrow time-of-flight slices (200-400 ns) of the elongated photofragment distribution are obtained by using a fast high-voltage switch (Behlke, 10 ns rise time) to gate the front MCP. Images are recorded using a free-running charge-coupled device camera (Proxitronic HR0, $768 \times 576$ pixels, $50 \mathrm{~Hz}$ repetition rate) and sent to a frame grabber. The output is averaged over a cycle of 20 frames, and each image presented is the summation of $\approx 300$ acquisition cycles.

Velocity mapping is provided by an Einzel lens mounted beyond the extractor grid. Satisfactory mapping is obtained for $V_{\text {Einzel }}$ greater than a minimum voltage, the magnitude of which is determined by $V_{R}$, but otherwise the lens voltage is not critically linked to the magnitude of $V_{\mathrm{R}}$. As an example, for $V_{\mathrm{R}}=1000 \mathrm{~V}$, a lens voltage of $V_{\text {Einzel }}>150 \mathrm{~V}$ is required for proper velocity mapping. This property of the Einzel lens enables it to be used as a magnifying lens with which to enlarge the image. A lens voltage of $V_{\text {Einzel }}=218 \mathrm{~V}$ is chosen to provide a suitable degree of magnification.

The main differences between the present setup and the slice-imaging method described by Kitsopoulos and co-workers $^{7}$ are as follows: First, a small discrimination field $(3.2 \mathrm{~V} / \mathrm{cm})$ is applied at the time of excitation in order to separate prompt ions from the Rydberg states, whereas in the ion-slicing experiments the excitation and subsequent delay before applying the pulsed extraction field are under fieldfree conditions. Second, it is necessary to use a longer delay $(4-5 \mu \mathrm{s})$ before pulsed-field extraction, compared to the 100 ns to $2 \mu$ s employed by Kitsopoulos, to allow sufficient time for the separation of prompt ions and Rydbergs to occur. Although the use of a larger discrimination field would decrease the time required for this separation it would also destroy the population of long-lived high- $n$ Rydberg states. The position of laser excitation is moved further back from the extraction grid in order to avoid the fragments leaving the extraction region in this delay time. It should also be noted that the kinetic-energy release for the system studied here is lower than in the systems studied by Kitsopoulos and co-workers, so that even for a conventional ion-slicing experiment, longer delays would be required to achieve equivalent fragment-cloud expansion. Finally, the longer delays used in the present experiments spread out the time-of-flight distribution somewhat more than in the experiments of Kitsopoulos so that the time gate used for slicing can be somewhat wider than used previously.

\section{RESULTS}

In this section we compare the images obtained using the conventional slice-imaging approach, with those obtained by the Rydberg-tagging/slice-imaging method. In order to make a fair comparison we use approximately the same extraction delays and applied voltages for both types of experiment, with the exception of the small discrimination field applied for the Rydberg tagging. The first probe laser was positioned on the $Q_{11} / P_{21}(25 / 2)$ transition during acquisition of all the images presented here. This transition was chosen to minimize the contribution to the signal from rotationally cold NO impurities in the $\mathrm{NO}_{2}$ sample. Conservation of energy dictates that NO fragments, which are predominantly formed by the process

$$
\mathrm{NO}_{2}+h \nu \rightarrow \mathrm{NO}\left(v^{\prime \prime}=0, J^{\prime \prime}=25 / 2\right)+\mathrm{O}\left({ }^{3} P_{2}\right),
$$

will have a velocity of $880 \mathrm{~m} \mathrm{~s}^{-1}$ for a photodissociation wavelength of $355 \mathrm{~nm}$. 

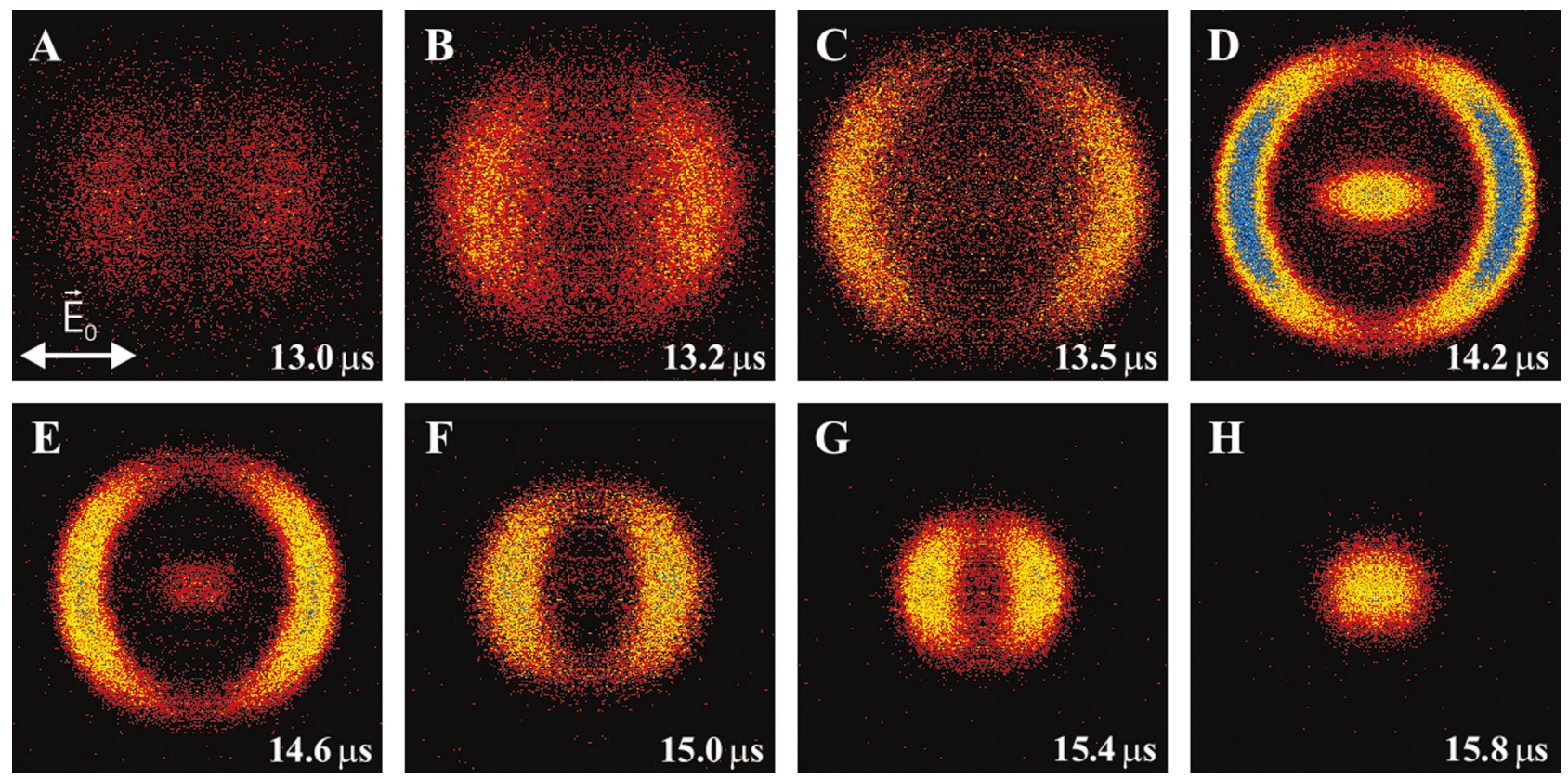

FIG. 2. (Color) $\mathrm{NO}^{+}$ion images obtained by gating different slices of the time-of-flight distribution which resulted from the photodissociation of $\mathrm{NO}_{2}$ at 355 $\mathrm{nm}$. The nascent NO fragments were ionized state selectively using a $\left(1+1^{\prime}\right)$ REMPI scheme. Image $D$ corresponds to the central slice of the distribution. The time at which the voltage pulse was applied to the detector is indicated in each panel. The images shown have been artificially symmetrized about the vertical axis.

\section{A. Conventional slice imaging of fragments ionized by REMPI}

Figure 2 shows the result of performing the conventional slice-imaging experiment on the nascent NO fragments, which are ionized by $\left(1+1^{\prime}\right)$ REMPI immediately after formation. Images were accumulated over 5000 laser shots, and correspond to $\sim 10^{4}$ ions detected. For an extraction delay $\tau=0$ the time-of-flight spectrum consists of a single NO peak of full width at half maximum $\sim 1 \mu \mathrm{s}$, but as $\tau$ is increased to $5 \mu$ s the peak broadens to a width of $3.5 \mu$ s. Slices (200 ns) of the elongated photofragment time-of-flight distribution [see Fig. 3(c)] were obtained by application of a fast $-400 \mathrm{~V}$ pulse to the front MCP. The fastest section of the ion cloud, corresponding to those ions which are initially backscattered relative to the molecular beam direction, is shown in image $A$ of Fig. 2. The subsequent images are recorded at progressively later arrival times. The images shown in Fig. 2 have been artificially symmetrized about the vertical axis in order to illustrate more clearly the changes in radial distribution across the series of images. The radius of the images reaches a maximum in image $D$, which corresponds to the slice through the center of the distribution. At later arrival times, the contribution from forward-scattered ions is recorded and the image radius becomes progressively smaller. Figure 2 demonstrates that despite the minor modifications to our setup in comparison to that of Kitsopoulos and co-workers, ${ }^{7}$ effective slicing can still be obtained. The fact that clean slicing is not observed for backscattered ions is possibly a result of inhomogeneities in the field in the vicinity of the $2 \mathrm{~mm}$ aperture in the repeller plate.

The feature in the center of images $D$ and $E$ is due to nonresonant ionization of rotationally cold NO impurities in the beam. This contribution is most significant in the central slices since it does not arise from a photolysis product and therefore does not spread significantly in the time-of-flight dimension. It is shown below that this "noise" is eliminated when Rydberg-tagging is utilized.

The center slice of the photofragment distribution (image $D$ of Fig. 2) is shown as a raw unsymmetrized image in Fig. 3(a), together with the time-of-flight profile [Fig. 3(c)], which indicates the width of this slice in comparison to that of the unsliced photofragment distribution. The slight asymmetry of the image can be attributed to the supersonic expansion not passing through the exact center of the Einzel lens. The speed distribution [Fig. 3(b)] can be determined from the center slice by applying the appropriate weighting to each pixel and integrating over $\theta$, as described in Ref. 7 . (This procedure is identical to that which would be applied to a conventional Abel-inverted image.)

It is immediately evident that the "low-velocity" tail which would arise from the projection of the entire photofragment sphere on to the detector is absent. This, coupled with the fact that an identical velocity distribution is obtained when the time slice is reduced to $100 \mathrm{~ns}$, is an indication that efficient slicing of the photofragment distribution has been achieved. However, individual contributions from the three channels corresponding to the spin-orbit states of the oxygen atom, all of which are energetically accessible at a photodissociation wavelength of $355 \mathrm{~nm}$, are not resolved.

The angular distribution for a given product channel is determined by

$$
I(\theta)=\sum_{r_{1}}^{r_{2}} N(r, \theta)
$$




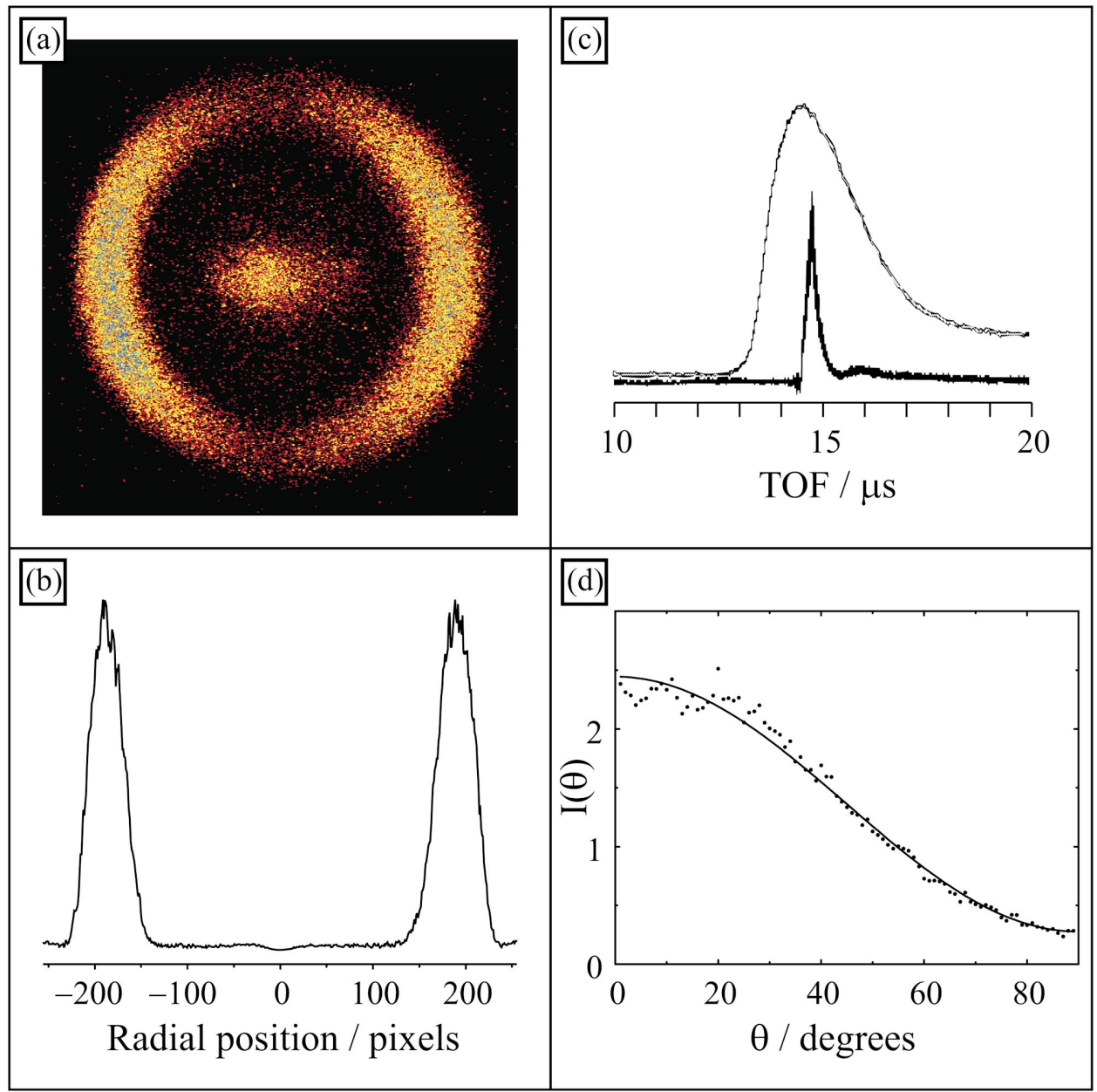

FIG. 3. (Color) (a) Central slice of the REMPI-detected ion images. (b) Radial distribution obtained from the image shown in (a); the width of the peak (43 pixels) corresponds to $200 \mathrm{~m} \mathrm{~s}^{-1}$ in true velocity space. (c) The overall time-of-flight profile (upper trace), and the gated signal (lower trace) corresponding to the image in (a). (d) The angular distribution extracted from the central slice (dotted line) and a fit of these data to Eq. (2) (solid line).

where $N(r, \theta)$ is the intensity of the slice image as a function of polar coordinates and $\theta$ is the angle between the photolysis laser polarization and the fragment recoil direction. $r_{1}$ and $r_{2}$ are radii chosen to encompass all the signal resulting from the channel of interest. To obtain a value for the anisotropy parameter, the angular distribution is fitted to the familiar expression for the spatial distribution of unpolarized photofragments, ${ }^{19,20}$

$$
I(\theta)=\frac{\sigma}{4 \pi}\left[1+\beta P_{2}(\cos \theta)\right]
$$

where $\beta$ is the anisotropy parameter, $\sigma$ is the total photofragment flux, and $P_{2}(\cos \theta)$ is the second Legendre polynomial. Figure 3(d) shows the experimental angular distribution determined from the center slice and averaged over all four quadrants, together with a simulation corresponding to $\beta$ $=1.44 \pm 0.04$. This value for the anisotropy parameter must be treated with caution since it is a summation of contributions from all three spin-orbit channels. However, it is in excellent agreement with the findings of Houston and co-workers ${ }^{21,22}$ who determined $\beta$ parameters for the dominant $\mathrm{NO}+\mathrm{O}\left({ }^{3} P_{2}\right)$ channel following the $355 \mathrm{~nm}$ photodissociation of $\mathrm{NO}_{2}$. Values in the range $\beta=1.2-1.6$ were obtained for various rotational states of $\mathrm{NO}\left(v^{\prime \prime}=0\right)$. This agreement provides confirmation that quantitative angular information can be simply extracted from slice images.

\section{B. Combining fragment Rydberg tagging and slice imaging}

Figure 4(a) shows a MATI (mass-analyzed-thresholdionization) spectrum recorded via the $A \leftarrow X(0,0)$ band of NO. The spectrum is obtained by fixing the first probe laser on the $Q_{11} / P_{21}(25 / 2)$ transition and scanning the second probe laser through the series of accessible ionization thresholds. Each peak corresponds to high- $n$ Rydberg states converging to an ionization threshold characterized by $N^{+}$, the ion core rotational quantum number. The spectrum was recorded using a $3.2 \mathrm{~V} / \mathrm{cm}$ discrimination field to reject prompt ions, and a pulsed extraction field of $F_{\text {ext }}=333 \mathrm{~V} / \mathrm{cm}$. The diabatic field ionization efficiency due to the extraction field is expected to drop from 1 at $E / \mathrm{cm}^{-1} \sim I-3.1 \sqrt{F_{\text {ext }}}$ to 0 at $E / \mathrm{cm}^{-1} \sim I-4.6 \sqrt{F_{\text {ext }}}$, where $I$ is the relevant ionization threshold (in $\mathrm{cm}^{-1}$ ) and $F_{\text {ext }}$ is the field (in $\mathrm{V} \mathrm{cm}^{-1}$ ). ${ }^{14}$ Therefore for $F_{\text {ext }}=333 \mathrm{~V} / \mathrm{cm}$ Rydberg states down to $\sim 80 \mathrm{~cm}^{-1}$ below threshold should be ionized. In practice, however, rapid sub-microseconds predissociation of low- $n$ Rydberg states restricts the range of $n$ that is observable and peak widths of order $\sim 20 \mathrm{~cm}^{-1}$ are observed. The high- $n$ Rydberg states are stabilized by $l$ - and $m_{l}$-mixing processes, ${ }^{14,23}$ and therefore lifetimes of the order of microseconds are observed. ${ }^{12}$ It is these stable high- $n$ Rydberg states which are crucial to the success of Rydberg-tagging experiments. 


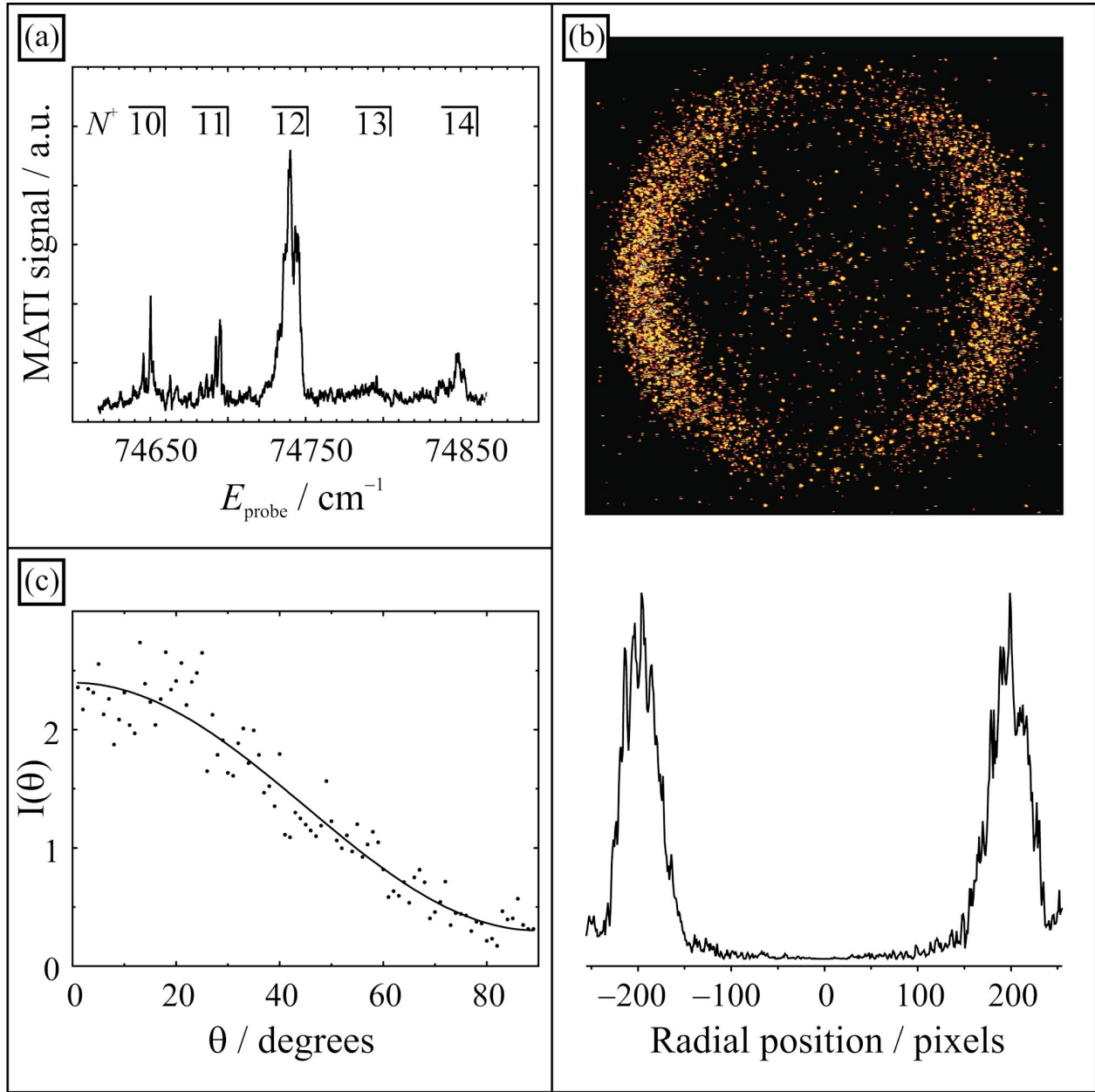

FIG. 4. (Color) (a) Mass-analyzedthreshold-ionization (MATI) spectrum of $\mathrm{NO}$ recorded by $\left(1+1^{\prime}\right)$ excitation through the $N^{\prime}=12$ levels of the $A^{2} \Sigma^{+}(v=0) \quad$ intermediate state; $E_{\text {probe }}$ is the total two-photon excitation energy. (b) Radial distribution (lower part of figure) obtained from the central slice of the Rydberg-tagged ion images (upper part); the width of the peak (42 pixels) corresponds to $190 \mathrm{~m} \mathrm{~s}^{-1}$ in true velocity space. (c) The angular distribution extracted from the central slice (dotted lines) and a fit to Eq. (2) (solid line).

As was discussed in Ref. 12, transitions with $N^{\prime}=N^{+}$ are favored and consequently the spectrum is dominated by the peak associated with the $N^{+}=12$ threshold. For the acquisition of the sliced images, the second probe laser was positioned at $n \approx 120$ of the pseudocontinuum below this threshold. Despite the relative intensity of the $N^{+}=12$ threshold in the MATI spectrum, signal levels obtained on this feature correspond to only $\sim 1 / 5$ of the $\left(1+1^{\prime}\right)$ REMPI signal just above threshold due to decay by Rydberg-state predissociation or autoionization.

The images obtained by the Rydberg-tagging technique are illustrated in Fig. 5 (again these have been artificially symmetrized about the vertical axis, as in Fig. 2, in order to show the general trend more clearly). Prompt ions are repelled by a $3.2 \mathrm{~V} / \mathrm{cm}$ discrimination field in the excitation region, and after a delay of $4 \mu$ s Rydberg-tagged fragments are field ionized and accelerated towards the detector by a $333 \mathrm{~V} / \mathrm{cm}$ field applied across the same region. A mapping voltage of $V_{\text {Einzel }}=218 \mathrm{~V}$ is used. Slices (400 ns) through the Rydberg-tagged photofragment distribution are shown, and these correspond to 5000 laser shots and $\approx 5000$ ions. For this series of images, wider slices were recorded (400 ns) than in the REMPI slice-imaging experiments described above, to ensure that sufficient signal levels were obtained at the extremes of the fragment cloud. Analogously to the REMPI slices, the images recorded at shorter arrival times correspond to the fragments that were originally backscat- tered with respect to the molecular beam direction. In the first image a contamination of the signal is observed due to the slight temporal overlap of the Rydberg-state and promption photofragment distributions at the detector. The appearance of the Rydberg-tagged slice images is, as would be expected, very similar to that of the corresponding REMPI slice images, with the important exception that the central noise arising from nonresonant ionization of $\mathrm{NO}$ is absent. The radii of the images increase with increasing time of flight until the central section of the photofragment distribution is reached (image $D$ ), and beyond this point the images decrease in size once more. The similarities between the ion and Rydberg-state results indicate that slice imaging is equally applicable to both REMPI and Rydberg-tagging detection schemes.

A 200 ns wide center slice of the Rydberg-tagged photofragment distribution is displayed (unsymmetrized) in Fig. 4(b), together with the corresponding radial distribution shown below. Once again, the intensity profile along the positive axis consists of a single unresolved peak. Significantly, the width of this feature is equal to that derived from the ion distribution of Fig. 3, demonstrating that the instrumental resolution is not reduced by the incorporation of PFI detection. The angular distribution derived from the center slice is well modeled assuming an anisotropy parameter of $\beta=1.4 \pm 0.2$, as shown in Fig. 4(c). This is consistent with both the figures obtained from the REMPI-detected ion im- 

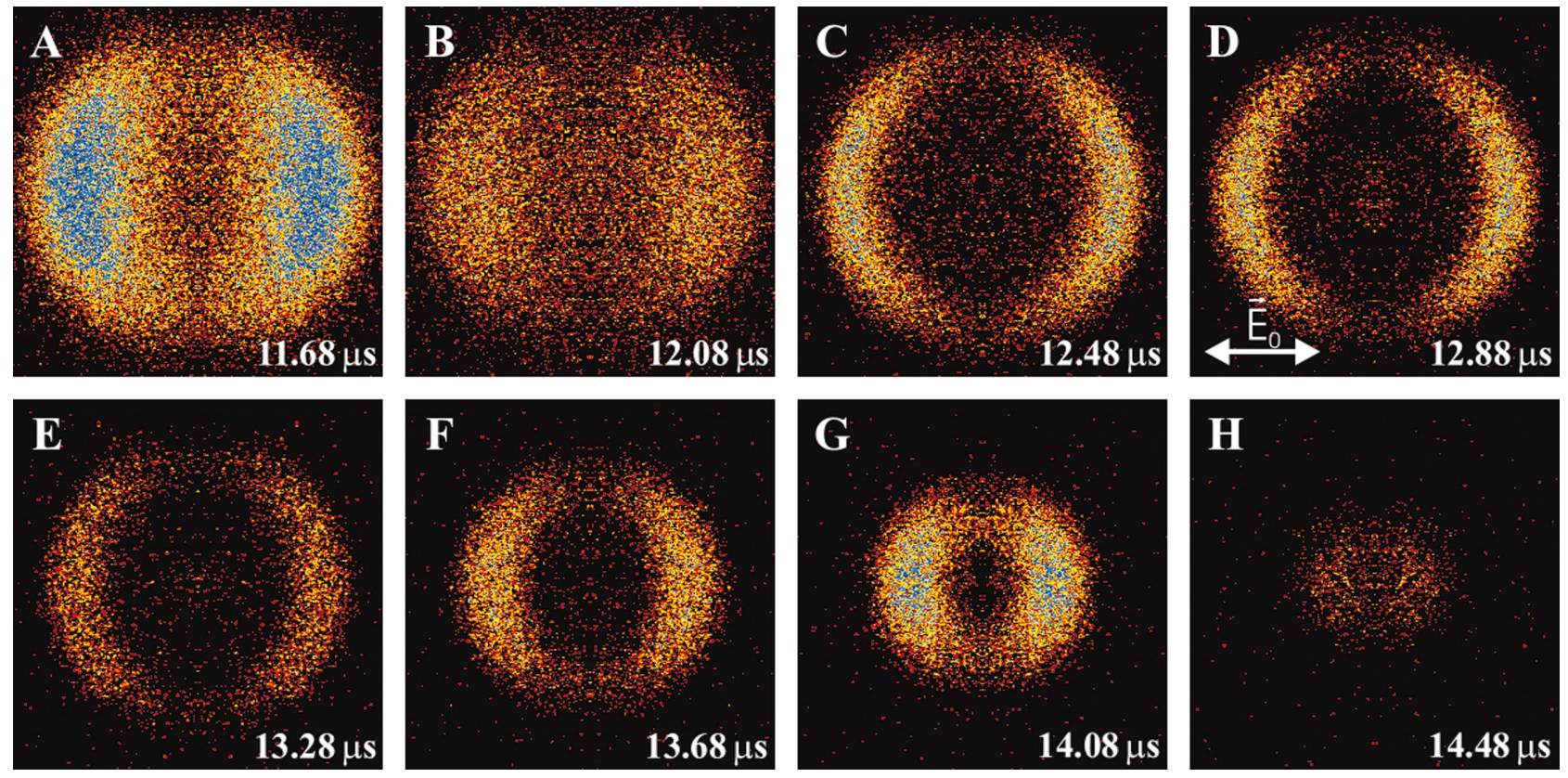

FIG. 5. (Color) $\mathrm{NO}^{+}$ion images obtained in the Rydberg-tagging/slicing experiments following photodissociation of $\mathrm{NO}_{2}$ at $355 \mathrm{~nm}$. These images were recorded by gating different slices of the time-of-flight distribution after delayed pulsed-field ionization of the Rydberg-tagged fragments produced by ( 1 $\left.+1^{\prime}\right)$ two-photon excitation. Image $D$ corresponds to the central slice of the distribution. The time at which the voltage pulse was applied to the detector is indicated in each panel. The images shown have been artificially symmetrized about the vertical axis.

age and the literature value, and is further proof of the compatibility of slice imaging and Rydberg-tagging techniques.

The breadth of the radial distribution peaks in Figs. 3 and 4 seems to imply that efficient velocity mapping has not been achieved in either the REMPI or the Rydberg-state experiments. The intention of using velocity mapping for photofragment trajectories is to reduce the blurring associated with the finite size of the laser interaction region. However, in the present case, it is unlikely that the velocity mapping provided by the Einzel lens will completely compensate for the large interaction region employed in these experiments. Thus, the use of crossed unfocused laser beams intersecting a free-jet expansion may be a limiting factor in terms of the achievable resolution. The image resolution of $\Delta E / E$ $\sim 20 \%$ is comparable to that obtained when detecting unmapped Rydberg-tagged fragments resulting from the interaction of focused lasers with a skimmed molecular beam (see, for example, the images shown in Fig. 4 of Ref. 12). This demonstrates that velocity mapping has at least partially compensated for the size of the interaction region and suggests that future experiments employing a skimmed expansion will yield a significant improvement in the resolution. In addition, in a slice-imaging experiment the quality of the velocity mapping is dependent on the dimensions of the photofragment cloud as it passes through the Einzel lens. Therefore, if the experimental conditions are chosen so as to yield a smaller cloud in this region then the photofragments will be more efficiently mapped, although this will be achieved at the expense of reduced resolution in the time-of-flight dimension. A reduction in the delay time $\tau$ will yield a smaller fragment distribution at the Einzel lens but achieving clean separation of Rydberg-tagged fragments and prompt ions becomes correspondingly more difficult. Alternatively, the slic- ing technique could be applied to threshold photodissociation studies, in which the rate of expansion of the fragment cloud is significantly reduced. As discussed earlier, the advantages of Rydberg-tagging methods are most evident in the nearthreshold region, making this a promising area of study.

A potential limitation of all slicing techniques is that the center slice is only equivalent to an Abel-inverted image in the limit of infinitesimally thin slicing; for slices of finite width the solid angle probed is dependent on the velocity of the fragments, and consequently slower fragments are detected with higher efficiency but lower velocity resolution. ${ }^{1}$ However, for the experiments presented here a limiting upper slice width can be found, below which the radial distribution of the image is independent of slice width. This is an indication that the variation of the solid angle probed with fragment velocity is so small as to have a negligible effect on the results.

Finally, it should be noted that despite the relatively low resolution currently obtained, the quality of the images is visibly superior to the images of Rydberg-tagged fragments previously recorded using other electrode configurations, ${ }^{12,13}$ and distortions due to grids are all but eliminated.

\section{CONCLUSIONS}

The results presented in this paper demonstrate that the incorporation of PFI detection into the slice-imaging methodology requires only minor modifications to the experimental procedure of Kitsopoulos and co-workers. ${ }^{7}$ Slice imaging of Rydberg states provides equivalent angular and velocity distributions to its ion-imaging counterpart, and will be applicable to most dissociation processes for which a MATI detection scheme can be used for one photofragment. In 
common with all Rydberg-tagging experiments, the lifetime of the Rydberg states limits the signal levels obtainable and this is an issue that needs to be considered. Improved MATI signals are commonly achieved using carefully designed field configurations to trap a high proportion of the Rydbergstate population in stable high- $l$ and $-m_{l}$ states. ${ }^{24}$ However, stabilization schemes need not be complicated; for example, it has been observed that the use of a pulsed discrimination field rather than a dc field can lead to an enhancement of the MATI signal. ${ }^{25-27}$

The work described here offers a methodology which will provide a complementary approach to current ionimaging techniques, and expand the number of chemical systems that are accessible for study. Although the velocity resolution in the present setup is limited by the size of the laser interaction volume and the dimension of the fragment cloud as it passes through the Einzel lens, we have demonstrated that the method is viable and produces valid results. We anticipate that, with some further optimization, the sliceimaging procedure will become the method of choice in circumstances where Rydberg tagging is the appropriate stateselective detection scheme for photofragment imaging. This will particularly be the case where REMPI spectroscopy of the photofragment is either unknown/poorly characterized (e.g., $\mathrm{NH}_{2}, \mathrm{C}_{2} \mathrm{H}_{5}$, singlet- $\mathrm{CH}_{2}$ ) or where intense focusing of UV lasers would be required to drive $2+1$ or $3+1$ REMPI processes via short lived intermediate states. Recent ZEKE (zero-kinetic-energy) photoelectron studies of free radicals using vacuum ultraviolet radiation ${ }^{18,28,29}$ have paved the way for the application of Rydberg tagging to a wide range of radical species.

${ }^{1}$ A. J. R. Heck and D. W. Chandler, Annu. Rev. Phys. Chem. 46, 335 (1995).

${ }^{2}$ E. A. Wade, K. T. Lorenz, J. L. Springfield, and D. W. Chandler, J. Phys. Chem. 107, 4976 (2003).
${ }^{3}$ A. T. J. B. Eppink and D. H. Parker, Rev. Sci. Instrum. 68, 3477 (1997).

${ }^{4}$ M. J. J. Vrakking, Rev. Sci. Instrum. 72, 4084 (2001).

${ }^{5}$ C. Bordas, F. Paulig, H. Helm, and D. L. Huestis, Rev. Sci. Instrum. 67, 2257 (1996).

${ }^{6}$ M. J. Bass, M. Brouard, A. P. Clark, and C. Vallance, J. Chem. Phys. 117, 8723 (2002).

${ }^{7}$ C. R. Gebhardt, T. P. Rakitzis, P. C. Samartzis, V. Ladopoulos, and T. N. Kitsopoulos, Rev. Sci. Instrum. 72, 3848 (2001).

${ }^{8}$ T. Kinugawa and T. Arikawa, J. Chem. Phys. 96, 4801 (1992).

${ }^{9}$ K. Tonokura and T. Suzuki, Chem. Phys. Lett. 224, 1 (1994).

${ }^{10}$ D. Townsend, M. P. Minitti, and A. G. Suits, Rev. Sci. Instrum. 74, 2530 (2003).

${ }^{11}$ T. P. Rakitzis, P. C. Samartzis, R. Toomes, L. Tsigaridas, M. Coriou, D. Chestakov, A. T. J. B. Eppink, D. H. Parker, and T. N. Kitsopoulos, Chem. Phys. Lett. 364, 115 (2002).

${ }^{12}$ O. L. A. Monti, H. Dickinson, S. R. Mackenzie, and T. P. Softley, J. Chem. Phys. 112, 3699 (2000).

${ }^{13}$ O. L. A. Monti, H. A. Cruse, T. P. Softley, and S. R. Mackenzie, J. Chem. Phys. 115, 7924 (2001).

${ }^{14}$ T. P. Softley, Int. Rev. Phys. Chem. 23, 1 (2004).

${ }^{15}$ M. N. R. Ashfold, I. R. Lambert, D. H. Mordaunt, G. P. Morley, and C. M. Western, J. Phys. Chem. 96, 2938 (1992).

${ }^{16}$ D. H. Parker, R. F. Delmdahl, B. B. L. G. Bakker, and H. P. Loock, J. Chin. Chem. Soc. (Taipei) 48, 327 (2001).

${ }^{17}$ S. Manzhos, H.-P. Loock, B. L. G. Bakker, and D. H. Parker, J. Chem. Phys. 117, 9347 (2002).

${ }^{18}$ S. Willitsch, J. M. Dyke, and F. Merkt, Helv. Chim. Acta 86, 1152 (2003).

${ }^{19}$ R. N. Zare and D. R. Herschbach, Proc. IEEE 51, 173 (1963).

${ }^{20}$ R. N. Zare, Mol. Photochem. 4, 1 (1972).

${ }^{21}$ T. Suzuki, V. P. Hradil, S. A. Hewitt, P. L. Houston, and B. J. Whitaker, Chem. Phys. Lett. 187, 257 (1991).

${ }^{22}$ V. P. Hradil, T. Suzuki, S. A. Hewitt, P. L. Houston, and B. J. Whitaker, J. Chem. Phys. 99, 4455 (1993).

${ }^{23}$ W. A. Chupka, J. Chem. Phys. 98, 4520 (1993).

${ }^{24}$ L. Y. Baranov, A. Held, H. L. Selzle, and E. W. Schlag, Chem. Phys. Lett. 291, 311 (1998).

${ }^{25}$ F. Merkt, J. Chem. Phys. 100, 2623 (1994).

${ }^{26}$ H. J. Dietrich, R. Lindner, and K. Müller-Dethlefs, J. Chem. Phys. 101, 3399 (1994)

${ }^{27}$ A. Held, L. Y. Baranov, H. L. Selzle, and E. W. Schlag, J. Chem. Phys. 106, 6848 (1997)

${ }^{28}$ S. Willitsch, A. Wuerst, and F. Merkt, Chimia 58, 281 (2004).

${ }^{29}$ S. Willitsch, J. M. Dyke, and F. Merkt, Mol. Phys. (to be published). 